\title{
THE QUALITY MANAGEMENT GROUP POSTER SESSION
}

P756

Benchmarking the Quality of Bone Marrow Collections and Monitoring Donor Safety by Reviewing the Average Decrease in Haemoglobin ( $\mathrm{Hb})$ Levels

S. Holtby ${ }^{1, *}$, R. Pawson ${ }^{2}$, A. Tailor ${ }^{1}$

${ }^{1}$ The London Clinic, London, ${ }^{2}$ NHSBT, Oxford, United Kingdom

Introduction: Our collection centre performs an average of 50 bone marrow harvests per year $(\mathrm{BMH})$ from altruistic healthy adult donors. BMH is recognized as a safe procedure but not entirely without risk of adverse events. There is a constant need to balance donor safety with recipient need and the increasing demands for larger donations. We decided to audit average Total Nucleated Cell counts (per ml) and benchmark against the National Marrow Donor Programme (NMDP) average. We also looked at the average drop in haemoglobin $(\mathrm{Hb})$ and 30 day recovery as part of JACIE standard CM 6.3.9 (follow up of donors).

Material (or patients) and methods: The medical assessment records and collection reports from 40 donors were reviewed and the $\mathrm{TNC} / \mathrm{ml} \times 10^{8}$ counts were calculated from the laboratory flow cytometry results. The data was also evaluated taking into consideration donor weight, gender, total number of requested cells vs collected cells and the total volume of BM harvested. An $\mathrm{Hb}$ level was taken 24 hours after BM donation. Results: From the sample of $40 \mathrm{BMH}$ 's (adult recipients) the average cell count of the collections was $0.177 \times 10^{8}$ TNC per $\mathrm{ml}$. The average volume of BM collected from our donors was $15 \mathrm{ml} / \mathrm{kg}$ (range $4.6-20 \mathrm{ml} / \mathrm{kg}$ ). The average drop in $\mathrm{Hb}$ was $37 \mathrm{~g} / \mathrm{l}$ (range 19-57) with post-op Hbs averaging $112 \mathrm{~g} / \mathrm{l}$ (range 83-147 g/l). 5 donors had post-op Hb of less than $100 \mathrm{~g} / \mathrm{l}$. In another case a male donor had given just $15.5 \mathrm{ml} / \mathrm{kg} \mathrm{BM}$ and yet his $\mathrm{Hb}$ dropped $56 \mathrm{~g} / \mathrm{l}$ from 180 to $124 \mathrm{~g} / \mathrm{l}$. The figure shows the relation between volume of BM collected plus drop in $\mathrm{Hb}$.

Conclusion: The average TNC per $\mathrm{ml}$ collected at our centre compares well with figures quoted in published literature $\left(0.16-0.22 \times 10^{8} \mathrm{TNC} / \mathrm{ml}^{1}\right.$ and by the NMDP $\left(0.183 \times 10^{8} \mathrm{TNC} / \mathrm{ml}\right)$.

NMDP quote that a $20 \mathrm{ml} / \mathrm{kg}$ BM collection drops the $\mathrm{Hb}$ by 40-50 g/l which could cause symptomatic anaemia. Our data showed most collections were under the NMDP standard (up to $20 \mathrm{ml}$ marrow/donor $\mathrm{kg}$ ) and smaller drops in $\mathrm{Hb}$ were seen.

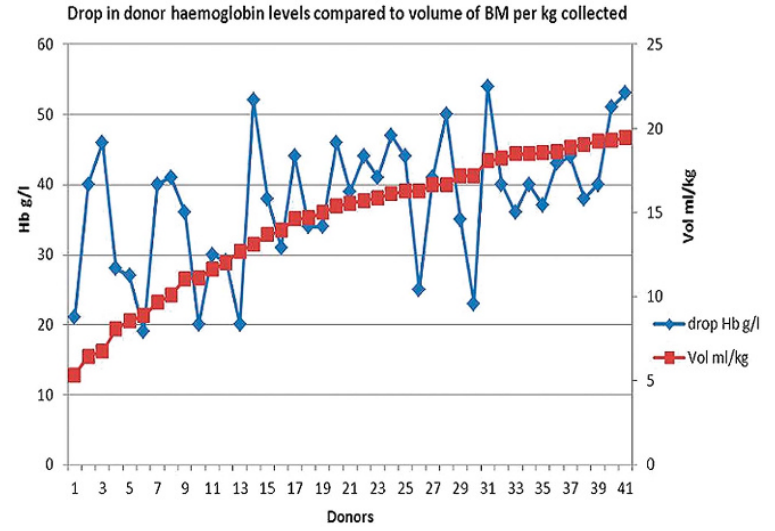

We conclude that we are achieving good cell counts from our donors and generally maintaining a post-procedure $\mathrm{Hb}$ of above $100 \mathrm{~g} / \mathrm{l}$. The information collected will help us inform donors about expected side effects such as fatigue and predict donors who may benefit from peri-operative iron supplementation. Data from day 30 blood counts will be reviewed to demonstrate recovery of $\mathrm{Hb}$ levels.

References: 1. T.-F. Wang et al. Biol Blood Marrow Transplant 17:351-355, 2011.

Disclosure of Interest: None declared.

P757

Reliability of Cyclosporine A Values taken through Central Venous Lines, - a Quality Management Study

A.-M. Berthelsen ", , H. Sengeløv ${ }^{7}$, K. H. Nørskov ${ }^{7}$, M. Nygaard ${ }^{1}$

${ }^{1}$ Hematology dept. BMT unit 4042, RIGSHOSPITALET, Copenhagen, Denmark

Introduction: HSCT patients are routinely treated with Cyclosporine A (CyA) post-transplant to prevent GvHD. CyA is administered orally or intravenously (IV).

We have experienced unexplainable high blood values in patients receiving oral or IV CyA. Normal practice is to draw blood samples from the CVC. Adherence of CyA to the catheter luminal wall is proposed as the cause during IV infusion and after discontinuing IV infusion of CyA.

A prospective study was performed in order to ensure the validity of CyA concentration in blood samples taken from peripheral vene and central venous catheters (CVC) and to define how long CyA is traceable after infusion of CyA in Hickmann catheter lumen.

Material (or patients) and methods: Myeloablative HSCT patients were recruited in the period from July 2014 to February 2015 at our bone marrow transplant center. All patients have given informed consent. CyA was analyzed concomitantly from: Peripheral blood (PB), CVC-line never used for CyA infusion (non CyA-Line) and CVC-line used for CyA infusion (CyA-line).

All samples from CVC were drawn according to our SOP, which includes discharge of the first $10 \mathrm{ml}$ blood drawn from CVC. All CVC's were three lumen Hickmann catheters.

Results: Patients $(N=8)$ participated in the study of CyA measurements from CyA-line, nonCyA-line and PB measurements. Results from blood samples drawn from CyA-line showed significantly higher values than from non CyA-line and $\mathrm{PB}$. CyA measurements from non CyA-line compared to PB values, showed no significant difference.

Patients $(N=8)$ participated in the study of CyA measurements after IV infusion. We measured CyA concentration from CyA-line, non CyA-line and PB. Result showed significant higher values for CyA-line after discontinuing the IV infusion compared non CyA-line and PB for at least two weeks after discontinuing IV CyA infusion.

Conclusion: During IV CyA infusion:

Blood samples drawn for CyA analysis from non-CyA-line are comparable to PB values. In contrast, CyA-measurements of blood drawn from CyA-line are significantly higher, despite use of the SOP procedure.

After IV infusion (CyA administered orally):

CyA values from non-CyA-line are in full correlation with values from PB. 
Up till two weeks after last infusion of CyA, blood samples from the CyA-line contain significantly higher levels of CyA. Even two weeks after discontinuing IV CyA there are differences in blood values.

During oral intake of CyA (control)

Measurements taken from a CVC-line, never used for IV infusion of CyA, compared to samples from $\mathrm{PB}$, are comparable for CyA.

We conclude that our practice of using the non-CyA-line for blood concentration measurements is justified. Use of the CyA-line for blood samples will produce incorrectly high values.

Importantly, falsely elevated CyA-values in the CyA-line are still obtained weeks after discontinuation of CyA infusion, which could lead to incorrect dose adjustment of CyA.

References: Elevated blood drug levels obtained from indwelling silicon catheters during oral cyclosporine $A$ administration.

A Claviez ${ }^{1}$, B Glass ${ }^{1}$, P Dreger $^{1}$ and M Suttorp ${ }^{2}$

${ }^{1}$ Bone Marrow Transplant Unit, University of Kiel, Kiel, Germany.

${ }^{2}$ University of Dresden, Germany.

Bone Marrov Transplantation March (2) 2002, Volume 29, Number 6, Pages 535-536.

Monitoring cyclosporine using blood drawn via a central venous catheter

J P Donnelly, N M A Blijlevens and A V M B Schattenberg, Nijmegen, The Netherlands.

Bone Marrow Transplantation (2003) 32, 1037.

Disclosure of Interest: None declared.

P758

Screen to screen contact for guiding stem cell transplant patient and caregiver at home: improvement of quality of life

H. Overbeek ${ }^{1, *}$, J. Vos ${ }^{1}, H$. Koene ${ }^{1}$ on behalf of outpatient team hematology

${ }^{1}$ Hematology, st Antonius Hospital, Nieuwegein, Netherlands

Introduction: After stem cell transplantation, patients normally stay at the hospital for two to three weeks until leucocyte recovery. For a selected group of hematology patients it is possible to spend the neutropenic phase after autologous stem cell transplantation at home. Crucial in this process is a 24 hour available caregiver. Some caregivers experience this as a heavy responsibility and a difficult task, accompanied by many uncertainties. We set up a system of ambulatory care in which patients have a hospital check three times a week supplemented with screen to screen contact via remote video technology. Because this is an innovative intervention, a feasibility study started in July 2015. The aim of this study is to evaluate the use of remote video technology in the home health care setting by judging the technical and visual quality as well as patient, caregiver and nurse satisfaction with this innovation. The study will end when saturation of the data occurs.

Material (or patients) and methods: Ten patients and their caregivers were recruited from the St. Antonius Hospital in Nieuwegein in the Netherlands. Patients were provided with a tablet and software. Wi-Fi at the patient's home was required. Twice a week the outpatient team set up a video call with the patient and caregiver, in addition to the ambulatory care provided three times a week. Data were sampled by keeping a logbook. Patient, caregiver and outpatient team outcomes were measured at the end of each session using semistructured questionnaires.

Results: All the patients and caregivers perceived both the tablet and communication application as easy to use. Ninety percent of the patients and caregivers were highly satisfied with the screen to screen contact with the ambulatory team. Ninety percent thought screen to screen contact had helped them feel more confident and safe in the ambulatory setting. Ten percent $(1 / 10)$ did not perceive any added value.
There were only two failed attempts $(2 / 45)$ to get in contact with patient and caregiver. In all other cases setting up the calls went smoothly. Sound and vision quality turned out to be dependent on the quality of $\mathrm{Wi}-\mathrm{Fi}$ at the patient's home. The image quality was fairly good, but not good enough to evaluate possible mucositis in the oropharynx. The outpatient team perceived great value to screen to screen contact for carrying out clinical eye assessment of the patient, and for its capability for clinical observation and the empowerment of both patient and caregiver.

Conclusion: The majority of patients and caregivers showed high satisfaction with the use of screen to screen contact. Both patients, their caregivers and the outpatient team appreciated screen to screen contact and the added value it gave to guiding patient and caregiver in the ambulatory care phase. The technical and visual quality of the screen to screen contact was experienced as good. The key advantages of this screen to screen contact, as seen by the outpatient team, were enhancement of increasing the patient's and caregiver's confidence while staying at home during the neutropenic phase.

Disclosure of Interest: None declared.

\section{P759}

Times and costs of unrelated donor search in italy: results from an analysis by the Italian Bone Marrow Donor Registry (IBMDR) and the Italian Group for Stem Cell Transplant (GITMO)

F. Bonifazi ${ }^{1,2, *}$ on behalf of GITMO, A. M. Gallina ${ }^{3}$, S. Guidi, S. Pollichieni ${ }^{3}$, F. Vagnozzi ${ }^{3}$, B. Bruno ${ }^{1}$, N. Sacchi $^{3}$ on behalf of IBMDR

${ }^{1}$ GITMO, IRCCS AOU San Martino - IST Istituto Nazionale per la Ricerca sul Cancro, Genova, ${ }^{2}$ Istituto di Ematologia L. e A. Seragnoli, Policlinico S. Orsola Malpighi, Bologna, ${ }^{3} I B M D R$, E.O. Ospedali Galliera, Genova, "Cattedra di Ematologia, A. O. di Careggi, Firenze, Italy

Introduction: One of the major limit of allogeneic transplants has been the availability of a suitable donor. In Italy the search of unrelated donors (UD) and cord blood units is pursued uniquely through Italian Bone Marrow Donor Registry (IBMDR); this is the only authorized Agency, which all Italian Transplant Centers refer to and its costs are completely paid by the public health care system through the National Registry (IBMDR).

A recent survey from the EBMT showed significant national differences in the attitude to use different type of donors. In Italy we observed a significant increase in the prevalence of haplos (28.2\% of allotransplants in 2014); we wondered if it could be explained by some specific criticalities in UD search in comparison to other Countries. The aim of the analysis was the evaluation of the probability and the time to find an UD and the costs of adult UD search in Italy.

Material (or patients) and methods: We analysed all the UD searches performed in Italy by 55 Centers from January $1^{\text {st }}$ to June $30^{\text {th }} 2015$.

Results: 881 searches have been initiated in favour of 879 patients affected by several haematological diseases. They requested 1684 samples on behalf of 746 pts. 1360 samples have been asked to three Registries (ZKRD -705-, IBMDR -331and NMDP -324-).1108 (65.8\%) samples arrived at the HLA labs with significant differences across the Registries $(75.5 \%$ German, 70.4 Italian, 44.4\% USA). 1091 samples have been finally typed and resulted at least 7/8 matched at 3, 6 and 10 months $81.9 \%, 89.3 \%$ and $90.6 \%$, respectively. The median time from search activation to donor identification was 37 days; the median time from search activation to verification typing was 5 days; the median time from verification typing to sample arrival at the HLA lab was 12 days and finally 16 days was the median time needed to perform the typing (HLA-A,B,C $\mathrm{DRB}^{*}$ and $\left.\mathrm{DQB} 1 * \mathrm{HR}\right)$. The 3 month-probability of finding a $8 / 8$ or at least $7 / 8$ matched donor has been 0.4 and 0.7 respectively. Only $41 . \%$ (369) of identified donors have been 
selected for work up and the median time from donor CT results to transplant has been 39 days $\left(1-3^{\text {rd }}\right.$ quartiles $\left.31-48\right)$. The overall cost of the procurement and shipment of the samples for confirmatory typing from adult donor have been 706.708,54 Euros. Significant differences have been found across the Registries as could be anticipated by the distance. The overall and median cost of the procurement of the samples have been 472.669 .40 and 427 Euros, while the overall and median cost of the samples transport have been 234039.14 and 211.23 Euros, respectively.

We observed a significant decrease of the cost of UD search in the last 3 years: the overall cost and mean cost per patient have been 21005282.82 and 10060.00 euros in 2012 vs 18968477.42 and 8901.21 euros, in 2014, respectively.

Conclusion: The results of this analysis showed that the efforts done by IBMDR on the re-organization of the complex nationally-scaled net involved in the UD search led to a significant reduction of the costs and improvement of the time to donor identification.

The shift to haploidentical transplant recorded in Italy cannot be attributed to inefficiency and costs of the UD search.

Disclosure of Interest: None declared.

\section{P760}

A Persistent Contaminant in a Cleanroom Environment Source, Elimination \& Prevention

W. Ogden ${ }^{1, *}$, C. Donohue ${ }^{1}$, P. Clarke ${ }^{1}$, R. Kettle ${ }^{1}$

${ }^{1}$ Stem cell Laboratory, CENTRAL MANCHESTER NHS

FOUNDATION TRUST, Manchester, United Kingdom

Introduction: Microbiological and physical monitoring of a clean room environment is an essential part of operational and performance qualification of a facility. All areas associated with aseptic preparation \& processing of therapeutic stem cell products must be assessed for compliance to appropriate standards applied to clean room facilities \& support areas (e.g. Rules and Guidance for Pharmaceutical Manufacturers and Distributors 2014 (Orange Guide).

At CMFT routine monitoring includes sessional, weekly, quarterly and annual tests as a minimum according to a layout agreed with Quality Control North West (QCNW). QCNW is a NHS regional specialty service providing comprehensive Quality Assurance Services. Alert, action and critical limits are set internally and where limits are exceeded, procedures describe actions to be taken.

During the first quarter of 2015 environmental monitoring results in the Stem Cell Laboratory at CMFT revealed out-of-limit growth for contact plates and finger dabs sampled in Grade A laminar flow cabinets; the contaminant (Bacillus organism) occurred sporadically but too frequently whilst settle plates indicated no growth in the same areas. The laboratory team put actions in place and the issue resolved for a short time but environmental results during April 2015 showed further episodes of contamination (Graph 1). The organism was eventually identified as swarming Paenibacillus lautus, an environmental contaminant that thrives in moist conditions.

Material (or patients) and methods: To try to identify the source of the contamination a detailed retrospective timeline was completed from December 2014. This allowed objective assessment of all reactive measures, the outcome and co-ordination of all results.

Results: The timeline indicated that the contaminant was being cleared efficiently but was consequently being re-introduced into the cleanroom environment.

Regular meetings were held to review results, cleaning procedures, biocidal cleaning product rotation strategy, enhanced screening, transfer validation of all consumables \& laminar air flow (LAF) efficiency. A peroxide vaprox decontamination of the whole unit \& personnel assessments were completed for compliance to all protocols. External experts were also consulted for advice (QCNW \& Product Specialist of Contamination Control).

We are unable to definitively confirm the exact source but there was strong evidence to suggest the cool packs used routinely in processing were the reservoirs and carriers for the contaminant.

- The same contaminant was isolated from the cool packs. No other item entering the laboratory grew the same organism when transfer validation tested.

- Due to the nature of the swarming bacillus (moisture loving) it was considered that the cool packs and associated condensation to be the source of the contamination.

- The cool packs are an essential item during processing and always present in the LAF cabinets.

- Both clean rooms (1\&2) had episodes of bacillus contamination; The cool packs are taken into both rooms.

Conclusion: New cool packs were introduced and are now subject to monthly environmental monitoring; a scheduled replacement is planned every six months at present.

Environmental Monitoring results have since remained ideal.

References: Rules and Guidance for Pharmaceutical Manufacturers and Distributors 2014 (Orange Guide).

Quality Control North West Guidance Documents.

Disclosure of Interest: None declared.

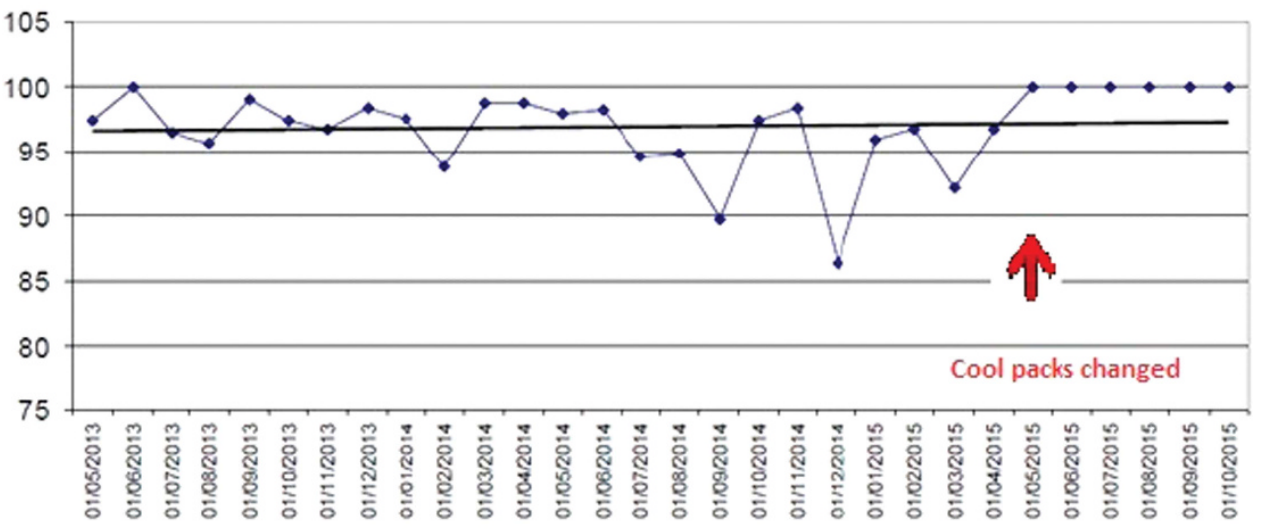

$\rightarrow \%$ Compliance $\longrightarrow$ Moving Average 
P761

Validation of microbiological sampling using automatic air sampler

E. Maytalman ${ }^{1}$, I. Kozanoglu ${ }^{1, *}$, C. Gereklioglu ${ }^{2}$, M. Unver ${ }^{1}$, S. Colakoglu, ${ }^{3}$, H. Ozdogu ${ }^{2}$, C. Boga ${ }^{2}$

${ }^{1}$ Cell Processing Unit, ${ }^{2}$ Clinical Unit, BASKENT UNIVERSITY ADANA ADULT BONE MARROW TRANSPLANTATION CENTER,

${ }^{3}$ Microbiology, Baskent University Medical Faculty, Adana, Turkey

Introduction: Microbiological controls done for air quality in critical areas is important for protecting the cellular product from microbial contamination and thereby the patients from infections during transplant process. Therefore air quality in critical areas should be periodically controlled with regard to bacterial and fungal contamination. Different techniques are used for microbiological measurements for air quality. The second samples were obtained using automatic air sampler (RGF Environmental, Florida/USA) since January 2015 in our center. The settle plates method is insufficient for quantitative assessment of the microbiological contamination in a certain air volume. The aim of this study is to determine whether there is a difference between conventional the settle plates method and automatic air sampler with regard to microbiological growing and to validate the new method for our center.

Material (or patients) and methods: Two separate samples were obtained from the critical areas and the devices in Cell Processing Unit using the settle plates method and air sampler. In the settle plates method, petri plates were left to air flow for 2 hours and samples were collected. Samples were obtained also using air sampler and all samples were transferred to Microbiology Laboratory for incubation.

Results: A total of six samples obtained monthly from the critical areas between January 2015 and June 2015 were evaluated for fungal and bacterial growing. Fungal and bacterial growing did not occur in the samples which were obtained using two methods in January, February and June. Fifty mold colonies were detected in the samples obtained from the incubator with each of two methods in March. One mold colony was detected in the sample obtained from the incubator using the settle plates method in April, in one of each samples obtained from the right of the clean room and quality laboratory in May however no colony was detected in the samples obtained using air sampler device. Bacterial growth was not detected in the samples obtained using both methods.

Conclusion: Petri plates are exposed to environmental factors in the settle plates method and this condition leads the other factors than air to increase contamination risk. Air sampler's yielding similar results with the manual technique and enabling quantitative assessment of colony counts suggested that this technique should be selected for microbiological air sampling.

Disclosure of Interest: None declared.

\section{P762}

"Slow and Steady" Cyclosporine Initiation is not associated with Increased Risk of Acute Graft Versus Host Disease - 12 Years' Experience From a Single Centre

A. E. Keegan ${ }^{1, *}$, Q. Tran ${ }^{2}$, P. Polistena ${ }^{1}$, J. Cooney ${ }^{1}$, P. Cannell ${ }^{1}$, M. Wright ${ }^{1}$, D. Purtill ${ }^{1}$

${ }^{1}$ Haematology, ${ }^{2}$ Pharmacy, Fiona Stanley Hospital, Murdoch, Australia

Introduction: There is much variation in clinical practice and little literature regarding the optimal dose and timing of cyclosporine (CSA) initiation for acute graft versus host disease (aGHVD) prophylaxis.

Material (or patients) and methods: To guide the development of consensus guidelines for CsA initiation at our centre, we reviewed information from consecutive recipients of allogeneic HSCT at our centre who received methotrexate and CSA GVHD prophylaxis and achieved neutrophil recovery between January 2004 and August 2015. Methotrexate was given at $15 \mathrm{mg} / \mathrm{m}^{2} \mathrm{IV}$ on day +1 and $10 \mathrm{mg} / \mathrm{m}^{2}$ on day $+3,+6$ and +11 . CsA was commenced at $1.5 \mathrm{mg} / \mathrm{kg}$ twice daily as an IV infusion and titrated to achieve a target trough level of $200-300 \mu \mathrm{g} / \mathrm{L}$, with routine levels taken every Monday, Wednesday and Friday.

Results: A total of 207 recipients of myeloablative $(n=171)$ and reduced intensity $(n=36)$ allogeneic HSCT were included. CsA was commenced on transplant day $-1(n=93)$ or earlier ( $n=109,5$ cases missing data) depending on physician preference. Antifungal prophylaxis was with fluconazole alone $(n=171)$ or another agent $(n=30,6$ cases missing data). Trough CsA levels are summarised in the Table. CsA levels ( $>200 \mu \mathrm{g} / \mathrm{L}$ ) were achieved in $12 \%$ of patients by day +3 and $42 \%$ of patients by day +5 , while $26 \%$ had low CsA levels $(<100 \mu \mathrm{g} / \mathrm{L})$ by day +3 and $5 \%$ by day +5 . The type of antifungal prophylaxis used did not correlate with the achievement of target CsA levels at any of the time points measured (Fisher's exact test). The cumulative incidence of grade II-IV aGVHD was $56 \%$ by day 180 (95\% confidence interval (Cl) 49-62\%). Neither the timing of CsA initiation (day -1 versus earlier) nor the CsA level by day +3 or day +5 , nor time to achieve target CsA levels were associated with the risk of aGHVD (Cox regression). Interestingly, patients with low $(<100 \mu \mathrm{g} / \mathrm{L}) \mathrm{CsA}$ levels by day +3 had a lower risk of grade II-IV aGVHD (HR $0.60,95 \% \mathrm{Cl} 0.38-0.95, P=0.029)$. On multivariate analysis adjusting for donor type (related versus unrelated) and conditioning regimen intensity, early CsA level $<100 \mu \mathrm{g} / \mathrm{L}$ was independently associated with a lower risk of aGVHD (HR 0.61, 95\% Cl 0.38-0.97, $P=0.035$ ).

\begin{tabular}{lcc}
\hline Trough CsA level & Median & Range \\
\hline $\begin{array}{l}\text { Time to achieve target } \\
\text { ment of CsA) }\end{array}$ & \\
$\geq 150 \mu \mathrm{g} / \mathrm{L}$ & 5 days & $1-342$ days \\
$\geq 200 \mu \mathrm{g} / \mathrm{L}$ & 8 days & $1-342$ days \\
& & \\
Trough CsA levels after stem cell infusion & \\
Day+0-3 & $127 \mu \mathrm{g} / \mathrm{L}$ & $34-594 \mu \mathrm{g} / \mathrm{L}$ \\
Day+3-5 & $187 \mu \mathrm{g} / \mathrm{L}$ & $58-435 \mu \mathrm{g} / \mathrm{L}$ \\
At engraftment & $255 \mu \mathrm{g} / \mathrm{L}$ & $78-561 \mu \mathrm{g} / \mathrm{L}$
\end{tabular}

Conclusion: At a uniform starting dose of $1.5 \mathrm{mg} / \mathrm{kg}$ twice daily IV, target CsA levels were only reached after a median 8 days of therapy. However, earlier achievement of target CsA levels did not infer a lower risk of aGVHD. Rather, patients with low CsA levels very early after transplant had a lower risk of aGVHD, independent of conventional GVHD risk factors. While this observation requires replication and further investigation, our analysis provides no rationale for more intensive initiation of CsA after allogeneic HSCT.

Disclosure of Interest: None declared.

P763

Time and Effort Quantification of Autologous Peripheral Blood Stem Cell Mobilization: A European Perspective K. Hubel 1,*, N. Azar ${ }^{2}$, L. Farina ${ }^{3}$, J. Reitan ${ }^{4}$, M. Gallagher ${ }^{5}$, M. Mohty

${ }^{1}$ U University Hospital of Cologne, Cologne, Germany, ${ }^{2}$ Service d'Hématologie Clinique, Hôpital de la Pitié-Sâlpétrière, Paris, France, ${ }^{3}$ Department of Hematology, Allogenic Bone Marrow Transplant Foundation, IRCCS National Institute for the Study of Cancer, Milan, Italy, ${ }^{4}$ RJM Group, Crown Point, ${ }^{5}$ Sanofi, Cambridge, United States, ${ }^{6}$ Saint-Antoine Hospital and University Pierre \& Marie Curie, Paris, France

Introduction: Autologous peripheral blood stem cell mobilization provides clinical benefit for improving stem cell collection prior to autologous or allogenic transplantation. 
With intense competition for hospital resources to evaluate and manage patients preparing for stem cell mobilization and transplantation, analysis of resource utilization is warranted. Plerixafor $(P)$ is indicated in combination with G-CSF to enhance mobilization of hematopoietic stem cells to the peripheral blood (PBSC) for collection, and subsequent autologous transplantation in patients with $\mathrm{NHL}$ and $\mathrm{MM}$ whose cells mobilize poorly; $\mathrm{P}$ has demonstrated significant impact on mobilization results.

Material (or patients) and methods: A non-interventional 2-part study using aggregated, de-identified data was conducted to analyze impact of plerixafor on efficiency of hematological department in hospitals associated with PBSC mobilization and apheresis in NHL patients in era prior to and following approval of plerixafor (to the "Pre- $\mathrm{P}$ " era through June 1, 2009, and " $P$ " era from July 1, 2010 onwards; "P", respectively). Adult patients $\geq 18$ years, with primary diagnosis of $\mathrm{NHL}$, who underwent PBSC mobilization at 9 European centers in France, Germany and Italy were included $(n=248)$. Part I: Retrospective medical record review of identified NHL patients. Outcomes measured in Part I included \# visits for administration of mobilizing agents and \# days administered; assessment of adverse events (AEs); \# apheresis sessions; hrs of apheresis sessions; attainment of CD34+ target; and days until CD 34+ target level met. For Pre-P era patients, peripheral CD-34+ count was recorded immediately prior to first apheresis; for $\mathrm{P}$ era patients, it was recorded immediately prior to plerixafor administration. Part II: Prospective time and motion evaluation of apheresis performed at each center; patient consent was obtained). Time-motion assessments, obtained retrospectively(Part I) \& prospectively(Part II), included total time to prepare patient, perform apheresis and manage AEs. Micro-costing was conducted by obtaining costs per unit of resource utilization from interviews with hospital staff. Study end points are difference in mean time/ effort to perform apheresis (including apheresis related AEs and total costs associated with mobilization to hospital between patients in Pre-P versus $P$ eras.

Results: No significant demographic differences were found between pre-P and $\mathrm{P}$ era cohorts. Analysis of \# apheresis sessions demonstrated statistically significant reduction $(P<0.001)$ for $P$ era group, including \# of sessions, total blood volume collected and total apheresis time required to reach targeted PBSC compared with Pre-P era group. Total cost differences between cohorts was estimated based on observed clinical and resource utilization differences. More than twice as many patients who had a CD 34+ $<10$ were mobilized in $\mathrm{P}$ era compared with patients in Pre-P era.

Conclusion: Final results of this study demonstrates that use of plexiafor is associated with statistically significant reductions in the \# apheresis sessions, driving both resource utilization efficiencies and cost savings for NHL patients undergoing autologous PBSC mobilization vs patients in pre-P era. Further research to quantify the time and motion and cost-consequences of treatment approaches for stem cell mobilization in routine clinical care is warranted to optimize treatment for NHL patients.

Disclosure of Interest: None declared.

\section{P764}

Managing Carbapenemase-producing Enterobacteriaceae

In A Transplant Setting

L. Hill, ${ }^{1, *}$, F. Dignan ${ }^{1}$, N. Blagburn ${ }^{1}$, M. Saif ${ }^{1}$, E. Tholouli ${ }^{1}$

${ }^{1}$ Clinical Haematology, CENTRAL MANCHESTER FOUNDATION TRUST, Manchester, United Kingdom

Introduction: Aim: To reduce/minimise the incidence of CPE acquisition within the Adult Stem Cell Transplant Unit at Manchester Royal Infirmary.

Background: Emerging multi-resistance bacteria is a rapidly increasing problem to healthcare both nationally and globally according Public Health England. Of all the Carbapenemaseproducing Enterobacteriae (CPE, CPC) may present the
CPE Acquisitions Ward 44 April 2014 to November 2015

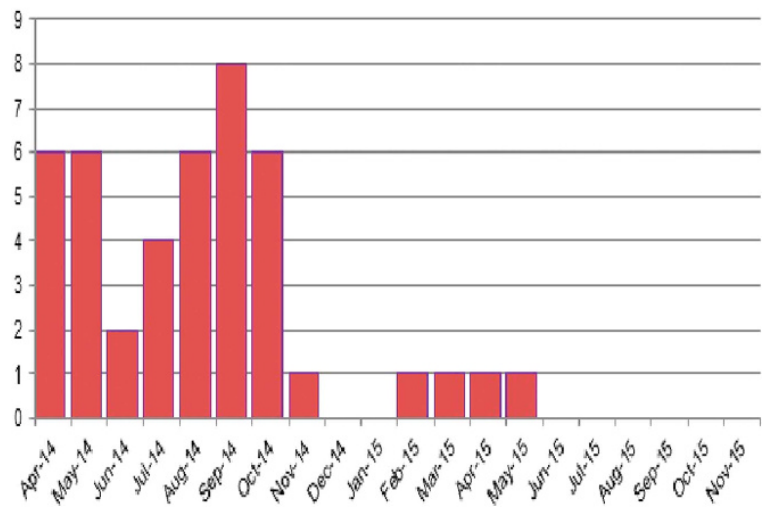

greatest risk to healthcare. Enterobacteriaceae are a large family of bacteria that usually live harmlessly in the gut of all humans and animals. Carbapenems are a valuable family of antibiotics such as Meropenem and imipenem normally reserved for serious infections caused by drug-resistant Gram-negative bacteria (including Enterobacteriaceae). Carbapenemases are enzymes that destroy carbapenem antibiotics, conferring resistance. At CMFT the first CPE was identified in 2009 with a steady increase year on year. From April 2014 the Adult Stem Cell Transplant Unit showed a significant increase in acquisitions leading to introduction of a weekly a screening programme for all in-patients from August 2014.

October 2014 a project was commenced to reduce the level of CPE colonisation within the Adult Stem Cell Transplant Unit by establishing a programme of hygiene interventions to prevent cross contamination between immune-compromised patients. Material (or patients) and methods: Review of previous service provision and cleaning schedules, daily MDT meetings with medical/nursing staff, infection control, microbiology and cleaning team. Environmental screening, decant \& deep clean of ward and implementation of change in practice with surveillance monitoring and audit. Introduction of cohort nursing practice.

Results: Environmental screening revealed contaminated dishwasher but no other contaminated areas were found. Introduction of new cleaning methods: infection clean followed by Deprox (regulated Hydrogen Peroxide vapour) of all ward areas during ward decant and after each patient discharge. Bay areas were converted to single occupancy initially reducing in-patient capacity from 30 to 16 beds. The single occupancy previously 4 bed bays were converted to 2 isolation rooms within the same space using a POD system, increasing the ward beds to 18 . All patients remain in isolation during their stay, shared patient day room closed, identified nursing and cleaning staff (cohort) to look after CPE carriers. Ward dishwasher was replaced. Introduction of long sleeve gowns when entering the room of a CPE carrier. Room specific Dynamap systems and cleaning equipment introduced including single use mop heads, sharps bins and equipment trays. Introduction of dedicated cleaning \& food service providers undergoing specialised training and continuous monitoring. Patient empowerment to report staff failing to comply with new policies. Strict hand hygiene practices introduced for all entering the ward (porters, visitors, allied health care proffesionals). Adherence to 2 visitors per patient per day.

Conclusion: A significant reduction in acquisitions was seen from November 2014; due to the large number of interventions introduced it has been difficult to identify a single intervention as being responsible for the dramatic decline in acquisitions. All the interventions remain in place with regular monitoring and review, it has now been 6 months since last acquision on ward 44

Disclosure of Interest: None declared. 
P765

Hypoglycemia during the temporary interruption of parenteral nutrition infusion in pediatric HSCT

R. Yanagisawa ${ }^{1,2,}$, K. Takeuchi ${ }^{3}$, K. Komori, ${ }^{1,2}$, I. Fujihara ${ }^{1}$, D. Morita ${ }^{2}$, M. Shiohara ${ }^{4}$, K. Sakashita ${ }^{1,2}$

${ }^{1}$ Department of Hematology/Oncology, Nagano Children's Hospital, Azumino, ${ }^{2}$ Department of Pediatrics, Shinshu University School of Medicine, Matsumoto, ${ }^{3}$ Department of General Pediatrics, Nagano Children's Hospital, Azumino, ${ }^{4}$ Department of Pediatrics, Matsumoto Dental University School of Dentistry, Shiojiri, Japan

Introduction: With the initiation of the conditioning regimen for HSCT, many patients experience difficulty in eating because of loss of appetite, nausea, and mucosal injury. Therefore, parenteral nutrition (PN) infusions are an important part of supportive care for such patients. Conversely, several drugs are required during the HSCT period, and in younger children, these require drip infusion because of the difficulty of oral intake. It is recommended that drugs such as acyclovir be diluted using only saline because the addition of other replacement fluids leads to many changes in these drugs. PN infusions have to be temporarily stopped to administer these drugs during HSCT. However, there have been no published data about complications arising from this situation. Material (or patients) and methods: The routine in our hospital included twice-daily (usually morning and evening) administration of acyclovir, starting in the morning at 7:00 AM for a 1-h drip infusion. Breakfast was served at 7:30 AM, and blood was usually collected before breakfast between 7:00 and 7:30 AM. Acyclovir was diluted with saline, and a saline flush was performed before and after the infusion to avoid change because of the mixing of acyclovir with another replacement fluid in the administration route. This daily medication and work routine for patients receiving HSCT continued in our hospital for 3 years. Therefore, we performed a retrospective analysis of 22 patients who received HSCT in those 3 years, examining their minimum blood glucose levels during the period from before the initiation of the preparative regimen to 30 days after HSCT.

Results: We divided the patients into two groups according to their minimum glucose levels during the period from before the initiation of the preparative regimen to 30 days after HSCT: minimum glucose level $<65 \mathrm{mg} / \mathrm{dL}$ (32-64, median $53 ; n=12$ ) and minimum glucose level $\geq 65 \mathrm{mg} / \mathrm{dL}$ (65-89, median 71 ; $n=10$ ). Using the Mann-Whitney $U$ test or Fisher's exact test, we compared the two groups in terms of several factors, including the day after HSCT the minimum blood glucose level developed, glucose infusion rate (GIR) as the minimum blood glucose level was developing, and each patient's background characteristics (age, gender, body weight, and body surface area). The minimum glucose level was significantly different between the groups $(P<0.0001)$, and the group that experienced hypoglycemia had a significantly higher GIR than the non-hypoglycemic group at the onset of the minimum blood glucose level $(P<0.0001)$. However, there were no other significant differences among the other factors. In the one of typical patient, a time-course analysis of blood glucose and immunoreactive insulin revealed a temporary PN infusion interruption before breakfast for acyclovir drip administration, and an infusion flush before drip induced hypoglycemia.

Conclusion: Particular attention may be required for pediatric patients when temporary PN infusion interruptions are needed regardless of their characteristics. The replacement fluid flush and drip line lock under high GIR also require special attention. Several precautions are needed to ensure safe pediatric practices, such as not interrupting PN infusions at times when hypoglycemia is more likely to develop, dilution with a $5 \%$ glucose solution when mixing drug infusions, slower flushing or locking, and ensuring glucose intake after PN interruption.

Disclosure of Interest: None declared. 